Original paper

\title{
Remnants of Early Carboniferous I-type granodiorite plutons in the Bavarian Forest and their bearing on the tectonic interpretation of the south-western sector of the Bohemian Massif (Bavarian Zone)
}

\author{
Fritz FINGER ${ }^{1 *}$, Daniel J. DUNKLEY², Miloš RENÉ ${ }^{3}$ \\ ${ }^{1}$ University of Salzburg, Division of Mineralogy, Hellbrunnerstrasse 34, A-5020 Salzburg, Austria; friedrich.finger@sbg.ac.at \\ ${ }^{2}$ National Institute of Polar Research, 3-10 Midoricho, Tachikawa, Tokyo-to, Japan 190-8518 \\ ${ }^{3}$ Academy of Sciences, Institute of Rock Structure and Mechanics, V Holešovičkách 41, 18209 Prague 8, Czech Republic \\ ${ }^{*}$ Corresponding author
}

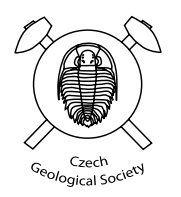

\begin{abstract}
The Bavarian Zone of the south-western Bohemian Massif is a late Variscan high heat-flow region, characterized by numerous granite intrusions and large amounts of LP-HT anatectic rocks, the latter comprising meta- and diatexites derived from sedimentary and igneous protoliths. Imaging and SIMS U-Pb dating of zircon from a distinctive type of hornblende-bearing diatexite, with a presumed igneous protolith, was done to gather information on both metamorphism and pre-anatectic crustal evolution. Zircon crystals have two phases of growth zoning, with oscillatory-zoned grains (visible through cathodoluminescence, CL, imaging) modified and surrounded by relatively uniform, high luminosity zircon. Analyses on oscillatory-zoned zircon yield an age of $344 \pm 2 \mathrm{Ma}(2 \sigma)$, interpreted as the formation age of the igneous protolith. More luminescent overgrowths, interpreted as having formed during anatexis, were dated at $323 \pm$ $3 \mathrm{Ma}$. Based on these dates, and on geochemical similarities, the protolith can be perfectly correlated with the high-K calc-alkaline Blatná suite of the Central Bohemian Plutonic Complex, $c .70 \mathrm{~km}$ to the north. This correlation strengthens tectonic models interpreting the Bavarian Zone as a reheated but integral constituent of the Variscan collisional crust of the Bohemian Massif, as opposed to an independent basement terrane assembled by late-Variscan orogenesis.
\end{abstract}

Keywords: Variscan orogen, Bohemian Massif, Bavarian Zone, Granodiorite, Zircon, U-Pb dating

Received: 20 September 2010; accepted: 2 December 2010; handling editor: J. Žák

\section{Introduction}

The Bavarian Zone of the south-western Bohemian Massif is an enigmatic high-T crystalline basement complex in the central European section of the Variscan collision orogen (Fig. 1). It is characterized by numerous granite intrusions and large amounts of LP-HT anatectic rocks, including meta- and diatexites derived from various metasedimentary and igneous protoliths. Recent work has shown that LP-HT metamorphism and granite plutonism in the Bavarian Zone occurred relatively late in the Variscan orogeny, between 330 and $315 \mathrm{Ma}$ (Finger et al. 2007; Siebel et al. 2008), at a time when adjacent regions in the central and eastern parts of the Bohemian Massif were already in a state of post-collisional exhumation and cooling (Fritz and Neubauer 1993; Urban and Synek 1995).

Because of the strong late Variscan regional metamorphism it is difficult seeing back into the earlier Variscan and pre-Variscan evolution of the Bavarian Zone, and it is controversially debated how to fit this zone into the plate tectonic puzzle of the Variscides. One concept is that major parts of the Bavarian Zone represent a fully independent plate tectonic terrane (Fiala et al. 1995; Siebel et al. 2008). A second model (Finger et al. 2007) presumes that the Bavarian Zone had a history common with the adjacent parts of the Bohemian Massif, before it became affected by the late Variscan heat flow.

A crucial point in this discussion is whether the Bavarian Zone contains lithologies that correlate with formations further north in the Moldanubian sector of the Bohemian Massif (see Finger et al. 2007; Finger and René 2009;

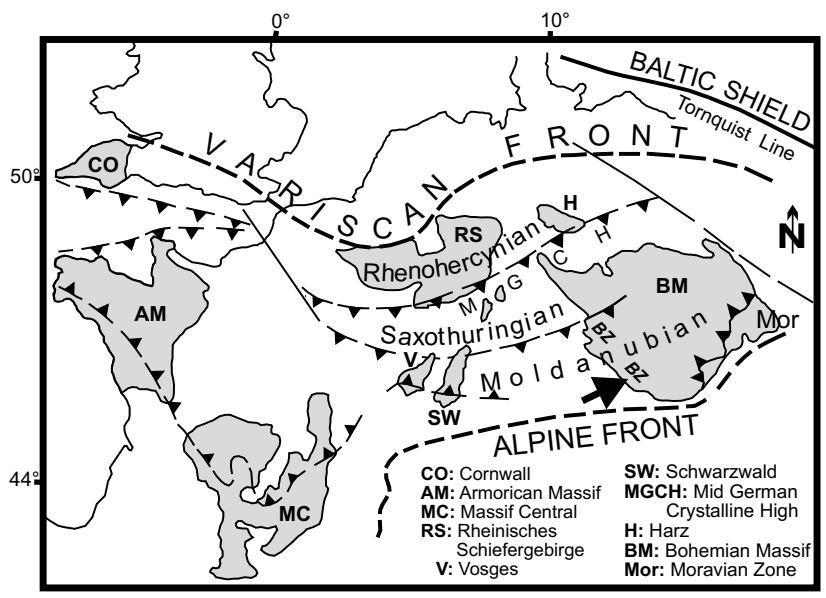

Fig. 1 Position of the Bavarian Zone (BZ) in the geological framework of Variscan Europe (map mainly after Franke 1989). 
Siebel et al. 2009). The problem is that such lithostratigraphic connections are difficult to prove, in view of the high-grade anatectic overprint of the Bavarian Zone. Based mainly on geochronological investigations, we will argue in this paper that the protolith of a hornblende-bearing diatexite, exposed near Waldkirchen in the Bavarian Forest, can perfectly be correlated with prominent, $c$. 340-350 Ma I-type plutonic activity in central Bohemia.

\section{Geological background}

The Variscan orogen is commonly interpreted as a collage of microplates (terranes) assembled between the Devonian and the Carboniferous along the southern margin of the Old Red Continent (Franke 2000; Friedl et al. 2000; Franke and Żelaźniewicz 2002; Winchester et al. 2002). The multiple terrane collisions resulted in the formation of several temporally and spatially distinct fold belts now straddling the sutures. In the central European section of the Variscan orogen, with the Bohemian Massif as the main exposure (Figs 1-2), at least three such fold belts can be distinguished. Northern areas (Rhenohercynian Zone, Northern Phyllite Zone, Mid German Crystalline High) record the final closure of the Rheic ocean and the Carboniferous collision of Variscan Europe with the Old Red Continent (Franke 2000; Oncken et al. 2000; Zeh and Gerdes 2010). In the central part of the Bohemian Massif (Teplá-Barrandian Unit), a c. 380 Ma old phase of deformation and regional metamorphism is documented and interpreted in terms of an early Variscan collision between the Saxothuringian and the Teplá-Barrandian domains (Zulauf 1997). Czech working groups tend to interpret this part of the Bohemian Massif as a long-lived Variscan active plate margin, developed above a subducted Saxothuringian ocean and passive margin, respectively (Konopásek and Schulmann 2005; Schulmann et al. 2009). The large Central Bohemian Plutonic Complex (Fig. 2) is considered in this model as subduction-related magmatic arc (Žák et al. 2005, in print). Magma generation above a westward dipping Moravo-Moldanubian subduction system has been considered by Finger et al. (2007, 2009).

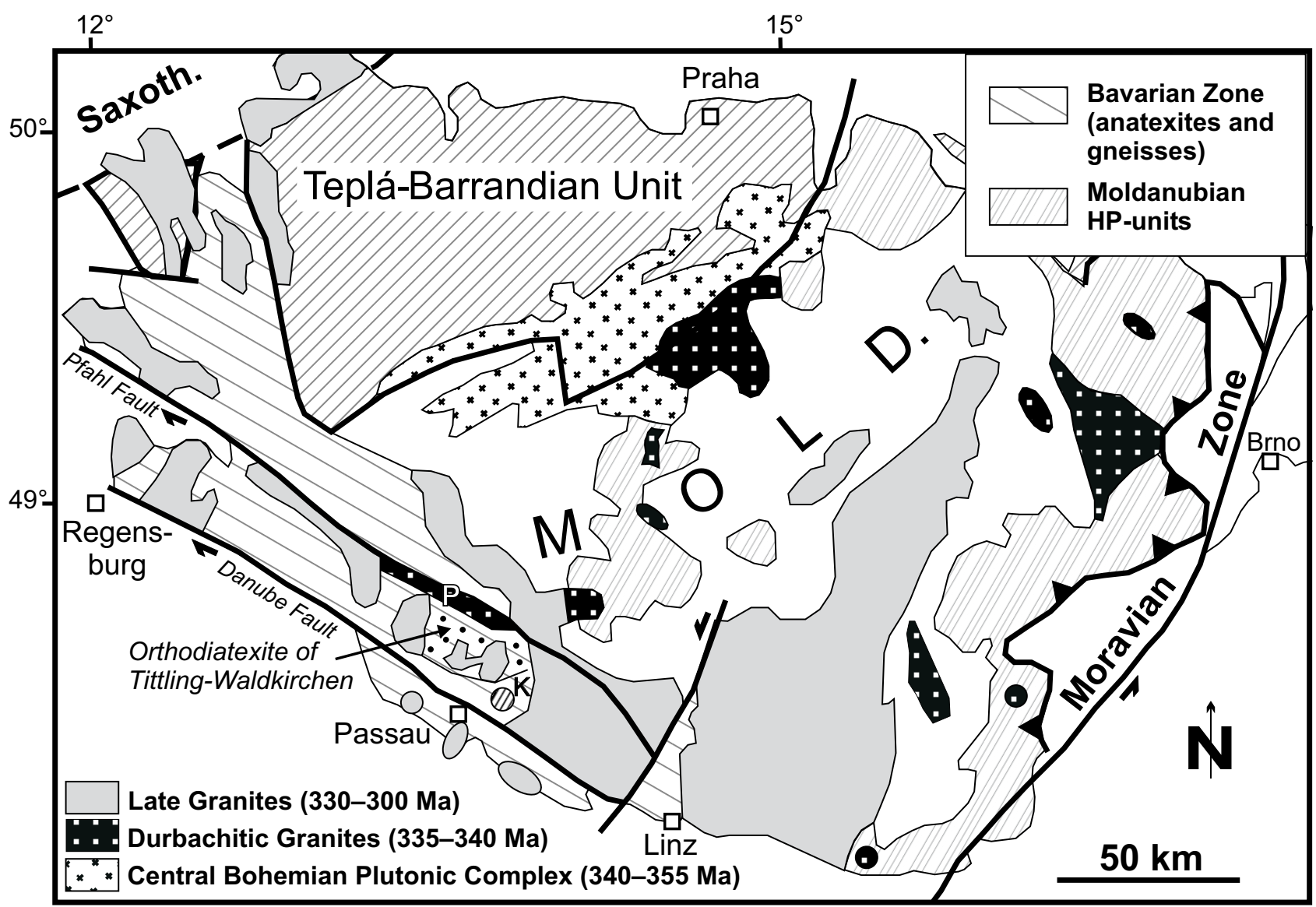

Fig. 2 Sketch map of the southern Bohemian Massif (from Dallmeyer et al. 1995, with minor modifications). Distribution area of the I-type orthodiatexite of Tittling-Waldkirchen according to Propach et al. (2008). P - palite (a potassic ortho-diatexite of durbachitic affinity), K - Kropfmühl Unit. See text for further explanation. 
In the south-eastern part of the Bohemian Massif, a major phase of collisional crustal thickening and highgrade regional metamorphism was recorded at $\sim 340 \mathrm{Ma}$, being related to the forceful docking of the Moldanubian and Moravian terranes (Finger and Steyrer 1995; Schulmann et al. 2005). After this "Moravo-Moldanubian" orogenic Phase (i.e. at c. $330 \mathrm{Ma}$ ), most of the Bohemian Massif was established in its present-day configuration (Edel et al. 2003). However, while large parts of the massif had arrived at a stage of post-collisional exhumation and cooling, the south-western sector (the Bavarian Zone - Fig. 2) was exposed to a prominent late Variscan tectonothermal event between 330 and $315 \mathrm{Ma}$, termed the Bavarian Phase of the Variscan orogeny (Finger et al. 2007). Large volumes of granitic rocks intruded this region and the country rocks were exposed to a penetrative high-grade regional metamorphism of the LP-HT type (Kalt et al. 2000; Tropper et al. 2006), which culminated in the formation of anatectic rocks (Thiele 1962; Fischer 1967; Troll 1967). In contrast to the generally NNE-SSW-striking collisional architecture preserved in the adjacent sectors of the Bohemian Massif, the Bavarian Zone received a completely new tectonometamorphic coining between 330 and 315 Ma with prominent NW-SE structures (Fuchs 1976; Stein 1988; Fiala et al. 1995).

Most anatexites of the Bavarian Zone are strongly peraluminous, biotite-rich, sillimanite and/or cordieritebearing rocks, and therefore most probably derived from (meta)pelitic protoliths. However, in places, feldsparrich anatexites of granitic or granodioritic composition can be found with probable igneous or orthogneiss protoliths (see Finger et al. 2007, for review). A large (c. $400 \mathrm{~km}^{2}$ ) occurrence of such "orthodiatexite" has been recently described by Propach et al. (2008) from an area $30 \mathrm{~km}$ NNE of Passau (Fig. 2). These ortho-diatexites of Tittling-Waldkirchen are medium- to coarse-grained, massive rocks rich in plagioclase (30-50\%), biotite (15-30\%) and quartz (10-20\%). Minor hornblende is commonly present. A typical feature of this diatexite is an abundance of fine-grained mafic enclaves and schlieren (Fig. 3). Hand specimens generally have a granitoid appearance and show only a weak foliation. However, on an outcrop scale, migmatitic textures can be recognized ubiquitously, from (1) layered variations in grain size and biotite content; (2) schlieren and patches of leucosome, and, most importantly; (3) the presence of swarms of mafic enclaves in various stages of arrested dissolution.

Based on geochemical investigations, Propach et al. (2008) interpreted the orthodiatexite of Tittling-Waldkirchen as mainly having formed through metamorphic/ anatectic recrystallization from volcanic (dacitic) protoliths, with mafic enclaves being remnants of basaltic-andesitic layers. However, this "volcanogenic" model is not the only possible scenario. Structures are equally compat-
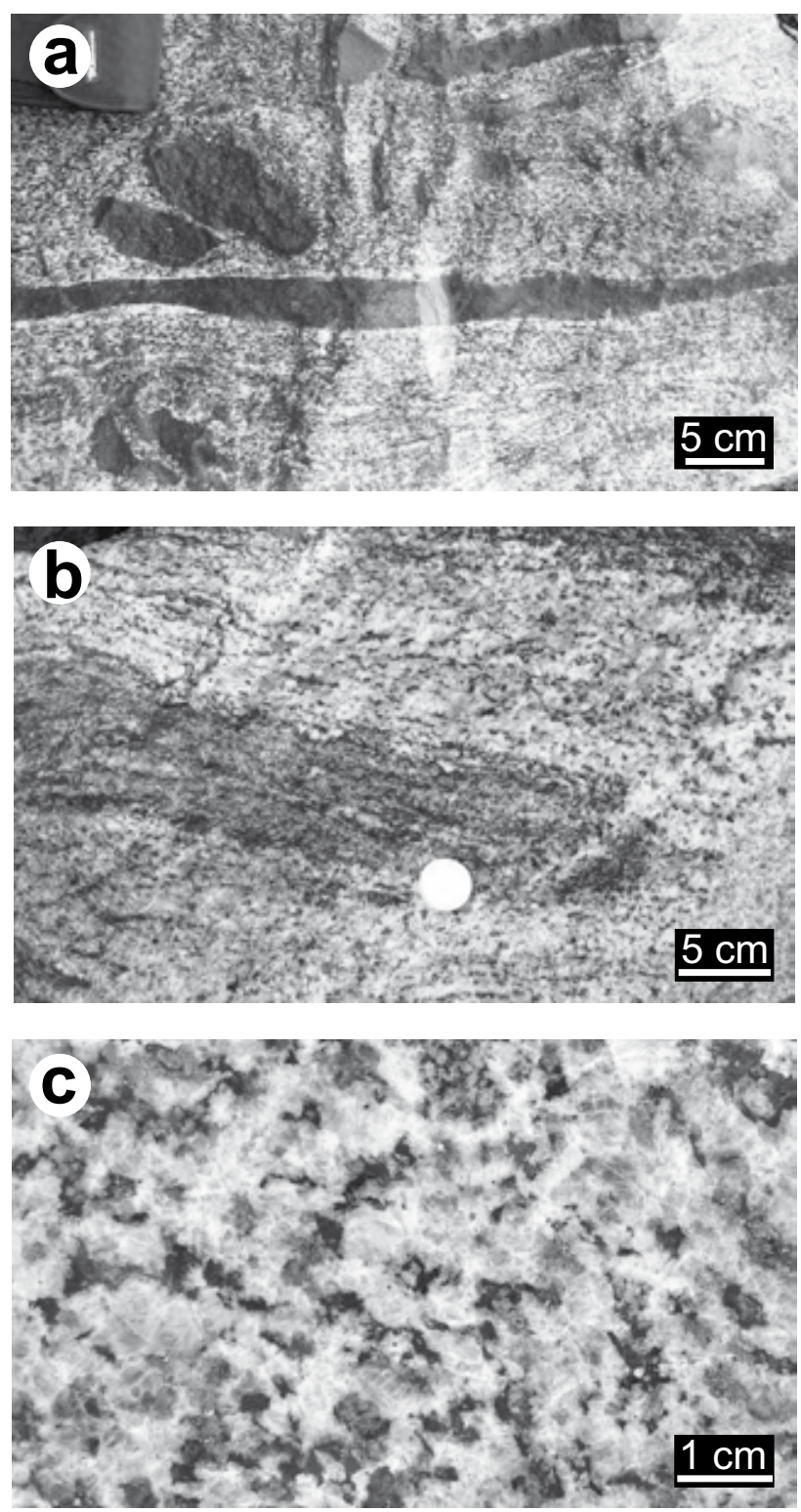

Figs 3 a-b Enclave textures in hornblende-bearing diatexite at a quarry in Steinerleinbach, probably resulting from former magma mingling processes in a granodioritic protolith. c - Typical coarse-grained Hblbearing diatexite (sample Fi14/07).

ible with a model that involves anatectic overprinting of a granodiorite intrusion containing dykes and enclaves of mafic magma. The shape, size and the swarm-like arrangement of the enclaves are comparable to those described from granite bodies elsewhere that were intruded by coeval mafic magma (Wiebe et al. 1997; Janoušek et al. 2000a; Słaby and Martin 2008). Notably, some of the dark enclaves include coarser granitoid material and resorbed feldspar crystals, characteristic of the mingling of mafic and felsic magmas. Examples can be found, where elongate mafic structures have an oblique angle to the main foliation implying an origin as discordant dykes. 
Furthermore, the diatexite contains pegmatitic portions that were deformed along with the host gneiss, suggesting a common pre-anatectic origin. These can be interpreted as relics of pegmatites intruded into a granitic host. Finer grained leucocratic portions of diatexite often follow elongate structures with an oblique angle to the main foliation and are reminiscent of pre-anatectic granite dykes.

\section{Objective and methods of the present study}

Due to the strong anatectic overprint, it is difficult to distinguish a plutonic versus volcanic protolith for the diatexite. However, geochronology of zircon permits a robust estimate of the time of magmatism (plutonic or volcanic), important for revealing regional correlations. $\mathrm{U}-\mathrm{Th}-\mathrm{Pb}$ isotope analysis of zircon by Sensitive HighResolution Ion Microprobe (SHRIMP) was performed, due to the isotopically robust nature of zircon during high-T metamorphism (e.g. Harley et al. 2007), and a sub-30 $\mu \mathrm{m}$ spatial resolution that allows discrete stages of zircon growth in individual grains to be dated.

About $10 \mathrm{~kg}$ of medium-grained Hbl-bearing diatexite from the Steinerleinbach quarry (sample Fi14/07 - Fig. $3 \mathrm{c}$ ) was crushed, sieved to $<500 \mu \mathrm{m}$ and processed on a wet shaking table to concentrate zircon. A selection of around 300 zircon crystals was mounted in resin and polished to grain mid-sections, cleaned to eliminate surface contamination by common lead, and coated with $100 \AA \AA$ of high-purity gold. The mount was imaged at high resolution by cathodoluminescence (CL) and electron backscattering (BSE) with a JEOL JSM-5900LV Scanning Electron Microscope (SEM) equipped with a Gatan MiniCL detector and JEOL SmileStation stage automation and image-capture software, at the National Institute of Polar Research (NIPR), Tokyo. Spots were carefully chosen from SEM imaging for analysis by SHRIMP-II at NIPR, using a primary $\mathrm{O}_{2}{ }^{-}$ion beam with a current of $2.5 \mathrm{nA}$ on the sample surface to produce a flat-floored oval pit, $25 \mu \mathrm{m}$ across. Secondary ionization was measured on a single electron multiplier on masses $196\left(\mathrm{Zr}_{2} \mathrm{O}\right)$ through to 254 (UO), with a mass resolution of $>5000$ for ${ }^{238} \mathrm{U}^{16} \mathrm{O}$ and a sensitivity on ${ }^{206} \mathrm{~Pb}$ of $>18 \mathrm{cps}$ per ppm per nA of primary current. Mass stations were measured through 6 cycles, including count times of $10 \mathrm{~s}$ per cycle for ${ }^{204} \mathrm{~Pb}$, background (at $204.04 \mathrm{amu}$ ) and ${ }^{206} \mathrm{~Pb}$, and $15 \mathrm{~s}$ for ${ }^{207} \mathrm{~Pb}$. Reduction of raw data for standards and samples was performed using the SQUID v.1.12a (Ludwig 2001) and Isoplot v.3.71 (Ludwig 2003) add-ins for Microsoft Excel 2003. Abundance of U was calibrated against zircon standard 91500 (80 ppm; Wiedenbeck et al. 1995). Corrections for common $\mathrm{Pb}$ on $\mathrm{U} / \mathrm{Pb}$ values and ages were done with common $\mathrm{Pb}$ estimated from
${ }^{204} \mathrm{~Pb}$ counts and the Stacey and Kramers (1975) common $\mathrm{Pb}$ model for the approximate $\mathrm{U}-\mathrm{Pb}$ age for each analysis. $(\mathrm{Pb} / \mathrm{U}) /(\mathrm{UO} / \mathrm{U})^{2}$ ratios were calibrated against 35 measurements on zircon standard TEMORA-2 (417 Ma; Black et al. 2004), with an error on the weighted mean of $0.41 \%(2 \sigma)$, and an external spot-to-spot error of $1.52 \%(2 \sigma)$; the latter was included in the errors on pooled Concordia ages (Ludwig 2003).

For geochemistry, a representative fresh slice was cut out from the sample $\left(c .300 \mathrm{~cm}^{3}\right)$ and milled to a fine powder in an agate mortar. The XRF measurements were carried out at Salzburg University on a Bruker S4 Pioneer instrument, equipped with a $4 \mathrm{~kW}$ Rh tube. Major elements were determined at reduced tube energies on glass beads ( $1 \mathrm{~g}$ sample : $4.5 \mathrm{~g}$ lithium tetraborate : $4.5 \mathrm{~g}$ lithium metaborate). Counting times were chosen in a way that the relative $2 \sigma$ uncertainties were better than $1 \%$ for $\mathrm{SiO}_{2}$ and $\mathrm{Al}_{2} \mathrm{O}_{3}$, and better than $5 \%$ for elements at the 1-10 wt. \% concentration level. For the determination of trace elements, which were measured on pressed powder pellets, counting times and tube conditions were optimized automatically up $4 \mathrm{~kW}$ and $400 \mathrm{~s}$ per element to obtain a detection limit of at least $3 \mathrm{ppm}(3 \sigma)$. Typical errors $(2 \sigma)$ from the counting statistics were $1-2 \mathrm{ppm}$ at low concentrations $(<10 \mathrm{ppm}), \sim 5 \mathrm{ppm}$ at the $100 \mathrm{ppm}$ concentration level, and better than $50 \mathrm{ppm}$ at the 1000 ppm level.

\section{Petrography and geochemistry}

The sample used for dating comes from the large active quarry at Steinerleinbach, $5 \mathrm{~km} \mathrm{~W}$ of Waldkirchen $\left(48^{\circ} 44^{\prime} 31^{\prime \prime} \mathrm{N}, 13^{\circ} 32^{\prime} 49^{\prime \prime} \mathrm{E}\right)$, and is representative of the medium-grained (average grain size $\sim 5 \mathrm{~mm}$ ), Hblbearing diatexite type, which is widespread in the area of Tittling-Waldkirchen (Fig. 3c). Modal contents are $40 \%$ plagioclase, $\sim 20 \%$ quartz, $\sim 15 \% \mathrm{~K}$-feldspar, $\sim 20 \%$ biotite and $\sim 5 \%$ amphibole. The plagioclase has a uniform An content of around 30. Larger euhedral plagioclase crystals show Carlsbad twinning and numerous tiny inclusions of K-feldspar (antiperthite) that are concentrated in the crystal centers or arranged in concentric rings, thus most probably copying a former igneous zoning pattern. However, no former igneous zoning in the An-content is preserved implying that the plagioclases were totally reequilibrated during the anatectic event. Smaller plagioclases $(1-3 \mathrm{~mm})$ show neither antiperthite inclusions nor Carlsbad twinning, and are less euhedral. They may represent new plagioclase grown from anatectic melt.

The K-feldspar (1-3 mm) shows weak perthitic exsolution. It is concentrated in small schlieren and thus probably represents a newly formed anatectic mineral. Quartz $(0.5-3 \mathrm{~mm})$ is always anhedral. Biotite is red brown, rela- 
tively large (3-5 $\mathrm{mm})$, but mostly with an anhedral outer shape in particular at contacts with the plagioclase. Small acicular rutile inclusions (sagenite) are common in the biotite. The hornblende, dull green, euhedral and up to $5 \mathrm{~mm}$ in hand specimen, exhibits strong recrystallization in thin section, and grains consist of a mosaic of fibrous secondary amphibole. Short prismatic apatite (0.1-0.4 $\mathrm{mm}$ ) is an ubiquitous accessory mineral. Occasionally, euhedral allanite crystals $(0.1-1 \mathrm{~mm})$ can be seen.

The geochemical analysis of the dated sample Fi14/07 basically fits to the data set published in Propach et al. (2008) for the ortho-diatexite of Tittling-Waldkirchen (Tab. 1). Major-element contents correspond to a metaluminous, high-K I-type dacite or granodiorite, respectively. Also, the trace-element spectrum shows all the characteristic features of a high-K, I-type magma, such as high $\mathrm{Ba}$ (1464 ppm), Sr (455 ppm), LILE/HFSE enrichment, a fractionated REE pattern with a negative $\mathrm{Nb}$ anomaly relative to Th and the LREE, when normalized to chondrite.

\section{Zircon}

The zircons of sample Fi14/07 are relatively large (100-300 $\mu \mathrm{m})$, normal-prismatic in shape (1/b 2-4) and mostly euhedral with prominent $\{100\}+\{101\}$ faces. Fine oscillatory zoning is typically visible under the optical microscope, along with abundant acicular inclusions of apatite. These features are characteristic of magmatic zircon, and there is little obvious evidence for modification during anatexis. However, detailed cathodoluminescence (CL) imaging of polished sections through zircon crystals reveals the presence of bright euhedral overgrowths, and patches of modification and replacement of oscillatory-zoned zircon, in almost every grain (Fig. 4). Overgrowths are rarely more than $30 \mu \mathrm{m}$ thick, and are best developed on pyramidal terminations. In most grains, the boundaries of oscillatory zoned zircon are rounded, and high-CL margins show weak concentric zoning around these boundaries (Fig. $4 \mathrm{c}-\mathrm{h}$ ). Some high-

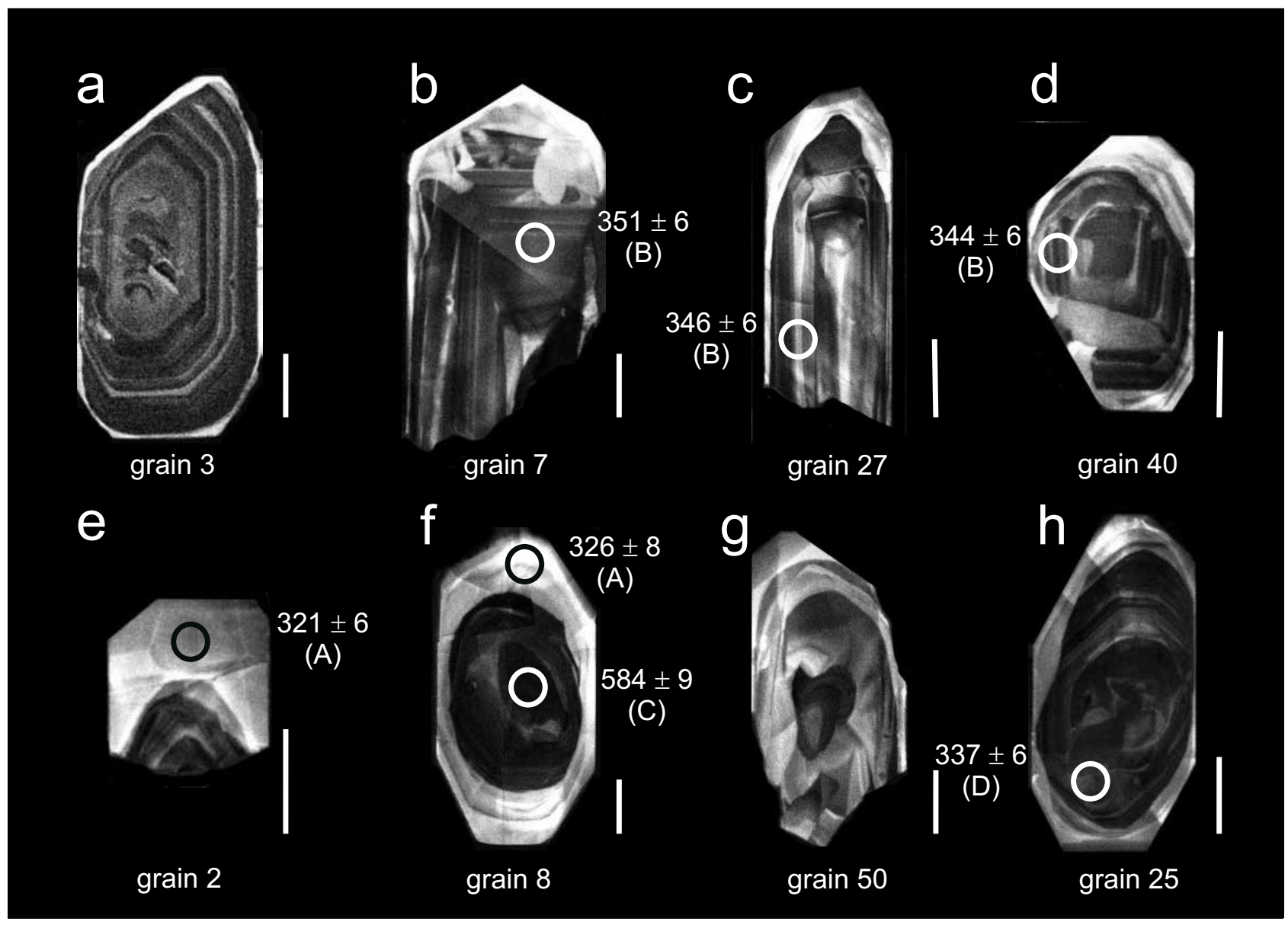

Fig. 4 Cathodoluminescence images of zircons from SHRIMP mount Fi14/07, showing characteristic microstructures as referred to in the text. a-d: grains with oscillatory zoned interiors (targets of group B analyses). These grains show variably thick overgrowths, partly truncating the previous growth zoning. e-f: zircons with particularly thick overgrowths (targets for group A analyses). Grain $\mathrm{f}$ contains an inherited inner core (C). $\mathbf{g}-\mathbf{h}$ : examples of zircons with convolute or patchy-zoned inner domains interpreted as partially recrystallized (group D analyses). Scale bar is always $50 \mu \mathrm{m}$. Shown are ${ }^{206} \mathrm{~Pb}-{ }^{238} \mathrm{U}$ ages $\left({ }^{204} \mathrm{~Pb}\right.$ corrected) with $2 \sigma$ errors (see Tab. 2 ). 
Tab. 1 Whole-rock geochemical (XRF) data for the dated sample Fi14/07

\begin{tabular}{|c|c|c|c|c|c|c|c|c|c|c|c|}
\hline & \multirow[t]{2}{*}{ Fi $14 / 07$} & \multicolumn{5}{|c|}{ Data set of Propach et al. (2008) } & \multicolumn{5}{|c|}{ Červená Granodiorite (René 1999) } \\
\hline & & Min & $\operatorname{Max}$ & Mean & \pm & $1 \sigma$ & Min & Max & Mean & \pm & $1 \sigma$ \\
\hline $\mathrm{SiO}_{2}$ & 62.97 & 55.24 & 64.68 & 59.62 & \pm & 3.44 & 60.16 & 66.62 & 63.00 & \pm & 1.84 \\
\hline $\mathrm{TiO}_{2}$ & 0.78 & 0.47 & 1.61 & 0.96 & \pm & 0.33 & 0.48 & 0.82 & 0.66 & \pm & 0.10 \\
\hline $\mathrm{Al}_{2} \mathrm{O}_{3}$ & 16.15 & 15.71 & 20.77 & 17.63 & \pm & 1.57 & 15.26 & 17.57 & 16.17 & \pm & 0.62 \\
\hline $\mathrm{Fe}_{2} \mathrm{O}_{3}$ & 5.33 & 4.76 & 9.73 & 6.70 & \pm & 1.69 & 3.77 & 5.55 & 4.79 & \pm & 0.57 \\
\hline $\mathrm{MnO}$ & 0.08 & 0.06 & 0.24 & 0.11 & \pm & 0.05 & 0.06 & 0.09 & 0.07 & \pm & 0.01 \\
\hline $\mathrm{MgO}$ & 3.35 & 2.42 & 4.05 & 3.15 & \pm & 0.51 & 2.09 & 3.44 & 2.80 & \pm & 0.44 \\
\hline $\mathrm{CaO}$ & 4.05 & 2.74 & 5.55 & 4.45 & \pm & 0.92 & 2.81 & 4.13 & 3.41 & \pm & 0.38 \\
\hline $\mathrm{Na}_{2} \mathrm{O}$ & 3.31 & 2.98 & 5.21 & 3.81 & \pm & 0.67 & 2.66 & 3.55 & 3.07 & \pm & 0.25 \\
\hline $\mathrm{K}_{2} \mathrm{O}$ & 3.35 & 1.47 & 3.91 & 2.92 & \pm & 0.83 & 3.48 & 4.48 & 3.88 & \pm & 0.31 \\
\hline $\mathrm{P}_{2} \mathrm{O}_{5}$ & 0.32 & 0.05 & 0.62 & 0.28 & \pm & 0.19 & 0.20 & 0.67 & 0.28 & \pm & 0.12 \\
\hline LOI & 0.71 & 0.25 & 1.90 & 0.90 & \pm & 0.39 & 0.64 & 2.01 & 1.10 & \pm & 0.44 \\
\hline Total & 100.40 & & & 100.52 & & & & & 99.22 & & \\
\hline $\mathrm{A} / \mathrm{CNK}$ & 0.98 & 0.94 & 1.21 & 1.01 & \pm & 0.08 & 0.98 & 1.12 & 1.05 & \pm & 0.08 \\
\hline $\mathrm{Ba}$ & 1464 & 978 & 1860 & 1324 & \pm & 335 & 893 & 1609 & 1331 & \pm & 185 \\
\hline $\mathrm{Rb}$ & 92 & 122 & 185 & 150 & \pm & 22 & 83 & 189 & 119 & \pm & 28 \\
\hline $\mathrm{Sr}$ & 455 & 348 & 576 & 464 & \pm & 82 & 261 & 466 & 374 & \pm & 60 \\
\hline $\mathrm{Y}$ & 21 & 5 & 38 & 18 & \pm & 13 & 11 & 41 & 27 & \pm & 7 \\
\hline $\mathrm{Zr}$ & 200 & 166 & 484 & 305 & \pm & 125 & 134 & 236 & 193 & \pm & 29 \\
\hline $\mathrm{Nb}$ & 12 & 8 & 31 & 15 & \pm & 9 & 8 & 18 & 13 & \pm & 2 \\
\hline Th & 9 & 2 & 17 & 7 & \pm & 6 & 7 & 22 & 15 & \pm & 5 \\
\hline $\mathrm{U}$ & 3 & n.d. & n.d. & n.d. & & & 2 & 12 & 7 & \pm & 3 \\
\hline $\mathrm{Ga}$ & 19 & 26 & 54 & 44 & \pm & 10 & 14 & 25 & 19 & \pm & 4 \\
\hline $\mathrm{Pb}$ & 22 & 14 & 24 & 18 & \pm & 4 & 24 & 57 & 41 & \pm & 12 \\
\hline $\mathrm{Zn}$ & 81 & 70 & 120 & 91 & \pm & 16 & 40 & 102 & 66 & \pm & 17 \\
\hline V & 95 & 69 & 184 & 119 & \pm & 37 & 40 & 100 & 68 & \pm & 19 \\
\hline $\mathrm{Ni}$ & 23 & 13 & 48 & 26 & \pm & 13 & 13 & 29 & 22 & \pm & 5 \\
\hline $\mathrm{Cr}$ & 58 & 31 & 113 & 65 & \pm & 32 & 26 & 124 & 67 & \pm & 32 \\
\hline $\mathrm{Sc}$ & 15 & 10 & 23 & 15 & \pm & 5 & n.d. & n.d. & n.d. & & \\
\hline Co & 14 & n.d. & n.d. & n.d. & & & 1 & 14 & 6 & \pm & 5 \\
\hline $\mathrm{La}$ & 34 & 23.0 & 60.0 & 37.8 & \pm & 15.6 & 23.3 & 62.5 & 41.3 & \pm & 16.6 \\
\hline $\mathrm{Ce}$ & 61 & 35.8 & 133.2 & 76.4 & \pm & 37.8 & 44.1 & 121.1 & 81.2 & \pm & 32.2 \\
\hline $\operatorname{Pr}$ & n.d. & 3.6 & 17.1 & 8.9 & \pm & 5.2 & 4.5 & 12.3 & 8.4 & \pm & 3.3 \\
\hline $\mathrm{Nd}$ & 36 & 12.3 & 68.9 & 34.4 & \pm & 21.2 & 16.5 & 45.3 & 31.3 & \pm & 11.9 \\
\hline $\mathrm{Sm}$ & n.d. & 1.9 & 13.7 & 6.3 & \pm & 4.4 & 3.0 & 9.2 & 6.4 & \pm & 2.5 \\
\hline $\mathrm{Eu}$ & n.d. & 1.5 & 1.9 & 1.7 & \pm & 0.2 & 0.9 & 1.5 & 1.2 & \pm & 0.2 \\
\hline $\mathrm{Gd}$ & 5 & 1.6 & 12.4 & 5.5 & \pm & 4.1 & 2.7 & 6.9 & 5.0 & \pm & 1.8 \\
\hline $\mathrm{Tb}$ & n.d. & 0.2 & 1.7 & 0.7 & \pm & 0.6 & 0.4 & 0.9 & 0.7 & \pm & 0.2 \\
\hline Dy & 3 & 1.2 & 7.9 & 4.0 & \pm & 2.7 & 1.9 & 4.7 & 3.7 & \pm & 1.3 \\
\hline Ho & n.d. & 0.2 & 1.4 & 0.8 & \pm & 0.5 & 0.4 & 0.8 & 0.7 & \pm & 0.2 \\
\hline $\mathrm{Er}$ & n.d. & 0.8 & 4.4 & 2.4 & \pm & 1.7 & 0.9 & 2.5 & 2.0 & \pm & 0.7 \\
\hline $\mathrm{Tm}$ & n.d. & 0.1 & 0.7 & 0.3 & \pm & 0.3 & 0.1 & 0.4 & 0.3 & \pm & 0.1 \\
\hline $\mathrm{Yb}$ & 2 & 0.9 & 4.9 & 2.2 & \pm & 1.8 & 0.8 & 2.6 & 1.8 & \pm & 0.7 \\
\hline $\mathrm{Lu}$ & n.d. & 0.1 & 0.8 & 0.3 & \pm & 0.3 & 0.1 & 0.4 & 0.3 & \pm & 0.1 \\
\hline
\end{tabular}

Data published by Propach et al. (2008) for the same orthodiatexite and data from the Červená granodiorite of the Central Bohemian Plutonic Complex (René 1999) are shown for comparison. 
CL rims also show sector-zoning that is discordant with oscillatory zoning in grain cores (Fig. 4e). These features are consistent with separate stages of zircon dissolution and subsequent overgrowth. However, other grains have more complex relationships between high-CL rims and cores (Fig. 4b) that are more characteristic of subsolidus modification (see Corfu et al. 2003).

The main parts of almost all zircon crystals have a lower CL response, and typically exhibit oscillatory zoning characteristic of growth in felsic magma (Fig. 4). In some grains, regular growth zoning is interrupted by convolute or patchy zoning with variable CL response (Fig. $4 \mathrm{~g}-\mathrm{h}$ ). Such convolute zoning can be magmatic, especially in the vicinity of inclusions, but may also be produced by modification of zircon by late or postmagmatic processes (Corfu et al. 2003). Few grains also preserve minor irregular and rounded cores, which are surrounded by oscillatory-zoned and/or relatively low-CL zircon (Fig. 4f, h).

\section{Results of SHRIMP zircon dating}

Forty-nine spot analyses were done on 41 grains (Tab. 2). Spots were selectively placed in texturally distinct zircon domains: (A) in high-CL overgrowths; (B) in the prevailing, oscillatory-zoned domains; (C) in rounded cores with zoning truncated by oscillatoryzoned zircon and (D) in domains with convolute or patchy zoning (examples in Fig. 4). The exact positions of analysis pits were checked after measurement with additional CL imaging by SEM. Four of them overlapped multiple domains, and were excluded (labeled 'Mix' in Tab. 2). Ten analyses with elevated common $\mathrm{Pb}$ contents $\left(>1 \%\right.$ of total ${ }^{206} \mathrm{~Pb}$ for $<1000 \mathrm{ppm} \mathrm{U}$, and $>0.5 \%$ for $>1000 \mathrm{ppm} \mathrm{U}$ ), which may yield unreliable age estimates, were also excluded (labeled " $X$ " in Tab. 2), as were two discordant analyses on overgrowths (grains 14 and 20).

Fig. 5 Terra-Wasserburg diagrams with group A (a) and group B (b) analyses $\left({ }^{204} \mathrm{~Pb}\right.$ corrected; errors are $2 \sigma$ ), including pooled Concordia ages (Ludwig 2003).
Group A analyses on high-CL overgrowths are characterized by $\mathrm{U}$ contents of $89-265 \mathrm{ppm}$ and $\mathrm{Th} / \mathrm{U}$ values between 0.48 and 0.82 . Together, 11 analyses define a Concordia age of $322.9 \pm 2.6 \mathrm{Ma}$ (95\% confidence errors; MSWD of equivalence $=0.98$; probability of concordance $=0.98$, plot in Fig. 5a). This age is interpreted as the time of zircon crystallization during anatexis.

Group B analyses on oscillatory-zoned zircon generally have higher $\mathrm{U}(312-763 \mathrm{ppm})$ and variably lower $\mathrm{Th} / \mathrm{U}$ $(0.23-0.63)$ than group A analyses. Together, 10 analyses define a Concordia age of $343.9 \pm 2.3 \mathrm{Ma}$ (MSWD of equivalence $=0.75$, probability of concordance $=0.55$, plot in Fig. 5b). We interpret this as the age of igneous protolith crystallization.

Three group $\mathrm{C}$ analyses scatter towards older ages, with the oldest being concordant at $c$. $584 \mathrm{Ma}$. These are interpreted as xenocrystic zircon incorporated into the protolith magma. Group D analyses, on zircon with
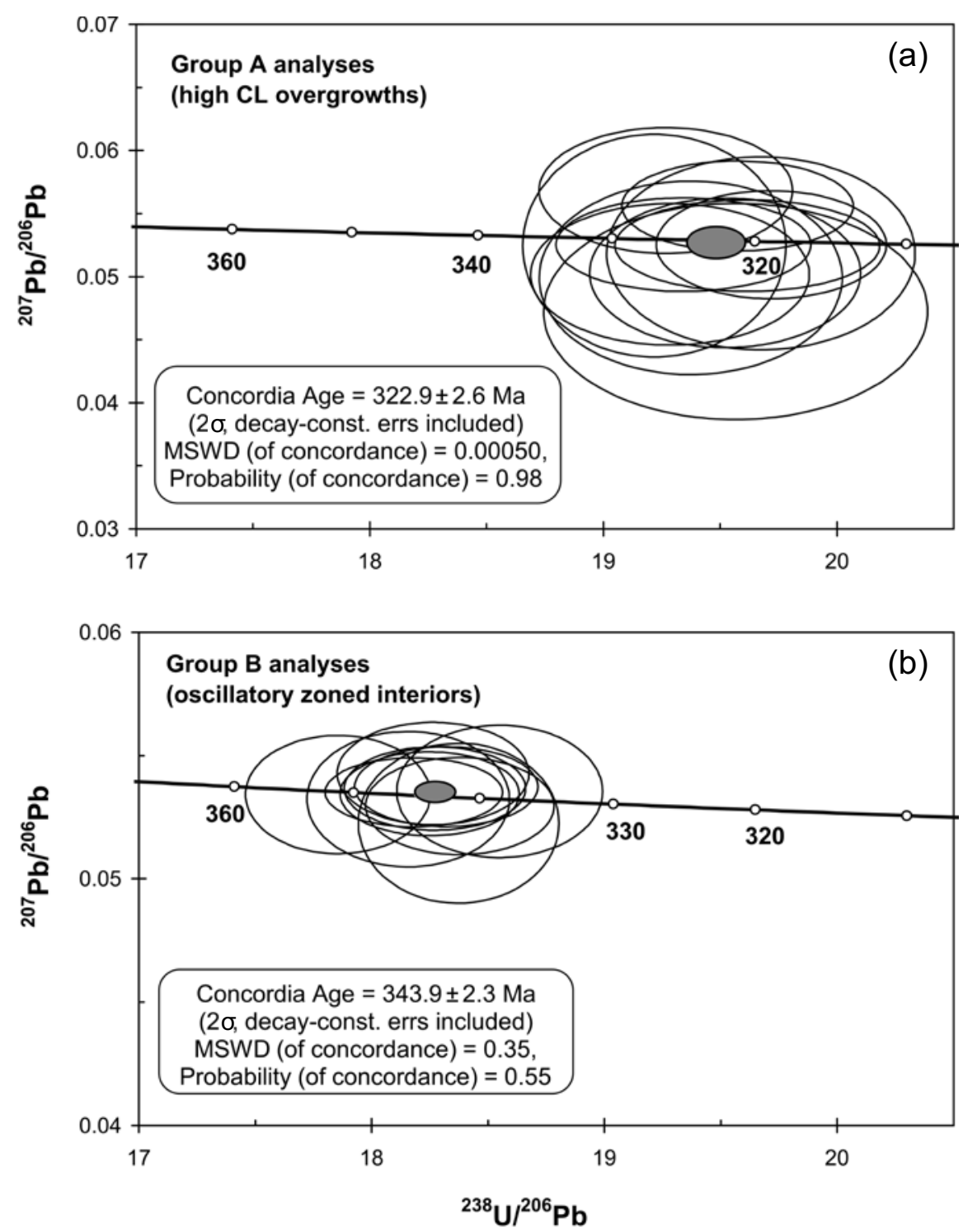
Tab. 2 Results of SHRIMP U-Pb zircon dating for sample Fi14/07

\begin{tabular}{|c|c|c|c|c|c|c|c|c|c|c|c|}
\hline Spot & Int. & $\begin{array}{c}\% \\
{ }^{206} \mathrm{~Pb}_{\mathrm{c}}\end{array}$ & $\begin{array}{c}\text { ppm } \\
\mathrm{U}\end{array}$ & $\begin{array}{c}\text { ppm } \\
\text { Th }\end{array}$ & ${ }^{232} \mathrm{Th} /{ }^{238} \mathrm{U}$ & $\begin{array}{c}\mathrm{ppm} \\
{ }^{206} \mathrm{~Pb}^{*}\end{array}$ & $\begin{array}{c}(1) \\
{ }^{206} \mathrm{~Pb} /{ }^{238} \mathrm{U} \\
\text { Age }\end{array}$ & $\begin{array}{c}(2) \\
{ }^{206} \mathrm{~Pb} /{ }^{238} \mathrm{U} \\
\text { Age }\end{array}$ & $\begin{array}{c}(3) \\
{ }^{206} \mathrm{~Pb} /{ }^{238} \mathrm{U} \\
\text { Age }\end{array}$ & $\begin{array}{c}(1) \\
{ }^{207} \mathrm{~Pb} /{ }^{206} \mathrm{~Pb} \\
\mathrm{Age}\end{array}$ & $\begin{array}{c}(1) \\
{ }^{208} \mathrm{~Pb} /{ }^{232} \mathrm{Th} \\
\text { Age }\end{array}$ \\
\hline Fi-14-07-01 & A & 0.78 & 90 & 54 & 0.62 & 4.0 & $319.6 \pm 4.3$ & $320.0 \pm 4.3$ & $319.8 \pm 4.8$ & $278 \pm 140$ & $318.0 \pm 16.0$ \\
\hline Fi-14-07-02 & A & - & 232 & 185 & 0.82 & 10.2 & $321.2 \pm 3.2$ & $320.1 \pm 3.3$ & $320.3 \pm 3.8$ & $436 \pm 58$ & $328.0 \pm 7.1$ \\
\hline Fi-14-07-04 & A & 0.63 & 107 & 69 & 0.66 & 4.7 & $321.3 \pm 5.4$ & $323.5 \pm 5.5$ & $323.8 \pm 6.0$ & $61 \pm 180$ & $298.0 \pm 15.0$ \\
\hline Fi-14-07-05 & $\mathrm{X}$ & 3.89 & 141 & 65 & 0.48 & 6.2 & $310.8 \pm 4.0$ & $314.0 \pm 3.6$ & $314.7 \pm 4.0$ & $-109 \pm 320$ & $259.0 \pm 30.0$ \\
\hline Fi-14-07-07 & $\mathrm{X}$ & 5.37 & 145 & 71 & 0.51 & 5.6 & $269.1 \pm 3.8$ & $270.4 \pm 3.3$ & $272.4 \pm 3.7$ & $75 \pm 350$ & $228.0 \pm 30.0$ \\
\hline Fi-14-07-07.2 & $\mathrm{B}$ & - & 506 & 238 & 0.49 & 24.3 & $351.3 \pm 3.1$ & $351.4 \pm 3.2$ & $350.8 \pm 3.4$ & $347 \pm 41$ & $357.7 \pm 6.1$ \\
\hline Fi-14-07-07.3 & $\mathrm{X}$ & 2.39 & 207 & 99 & 0.49 & 8.3 & $288.5 \pm 3.2$ & $288.5 \pm 3.1$ & $290.8 \pm 3.7$ & $293 \pm 170$ & $260.0 \pm 22.0$ \\
\hline Fi-14-07-08 & $\mathrm{X}$ & 1.67 & 115 & 70 & 0.63 & 4.6 & $288.3 \pm 3.8$ & $288.9 \pm 3.6$ & $292.8 \pm 4.0$ & $213 \pm 210$ & $245.0 \pm 18.0$ \\
\hline Fi-14-07-08.2 & A & 0.44 & 113 & 53 & 0.48 & 5.1 & $325.9 \pm 4.0$ & $327.0 \pm 4.1$ & $326.9 \pm 4.4$ & $203 \pm 110$ & $313.0 \pm 14.0$ \\
\hline Fi-14-07-08.3 & $\mathrm{C}$ & 0.03 & 1007 & 111 & 0.11 & 82.1 & $584.3 \pm 4.7$ & $584.5 \pm 4.8$ & $584.6 \pm 4.7$ & $573 \pm 17$ & $565.0 \pm 17.0$ \\
\hline Fi-14-07-11 & A & 0.42 & 143 & 71 & 0.51 & 6.3 & $322.0 \pm 3.8$ & $323.1 \pm 3.9$ & $320.5 \pm 4.2$ & $208 \pm 110$ & $340.0 \pm 13.0$ \\
\hline Fi-14-07-11.2 & B & - & 312 & 109 & 0.36 & 14.4 & $338.5 \pm 3.2$ & $338.3 \pm 3.3$ & $338.8 \pm 3.4$ & $352 \pm 46$ & $332.9 \pm 8.9$ \\
\hline Fi-14-07-12 & Mix & 0.44 & 281 & 93 & 0.34 & 12.9 & $334.0 \pm 3.2$ & $335.1 \pm 3.3$ & $336.8 \pm 3.4$ & $223 \pm 68$ & $281.0 \pm 11.0$ \\
\hline Fi-14-07-12.2 & $\mathrm{D}$ & - & 304 & 100 & 0.34 & 14.0 & $336.9 \pm 3.2$ & $337.0 \pm 3.2$ & $337.3 \pm 3.4$ & $330 \pm 41$ & $329.5 \pm 8.1$ \\
\hline Fi-14-07-13 & A & 0.40 & 89 & 54 & 0.62 & 4.0 & $324.4 \pm 4.3$ & $325.6 \pm 4.3$ & $323.7 \pm 5.0$ & $191 \pm 150$ & $332.0 \pm 19.0$ \\
\hline Fi-14-07-13.2 & $\mathrm{B}$ & 0.23 & 324 & 96 & 0.31 & 15.2 & $341.6 \pm 3.2$ & $342.1 \pm 3.2$ & $341.9 \pm 3.3$ & $297 \pm 58$ & $336.0 \pm 12.0$ \\
\hline Fi-14-07-14 & $\mathrm{X}$ & - & 117 & 64 & 0.57 & 4.9 & $309.0 \pm 3.8$ & $306.7 \pm 3.7$ & $308.0 \pm 4.1$ & $552 \pm 110$ & $320.0 \pm 16.0$ \\
\hline Fi-14-07-15 & $\mathrm{A}$ & 0.00 & 127 & 63 & 0.51 & 5.6 & $320.5 \pm 3.7$ & $320.7 \pm 3.8$ & $320.9 \pm 4.1$ & $305 \pm 64$ & $315.8 \pm 9.6$ \\
\hline Fi-14-07-16 & $\mathrm{X}$ & 1.75 & 184 & 139 & 0.78 & 8.1 & $314.3 \pm 3.6$ & $314.8 \pm 3.4$ & $318.2 \pm 3.9$ & $248 \pm 160$ & $284.0 \pm 13.0$ \\
\hline Fi-14-07-17 & A & 0.54 & 150 & 94 & 0.65 & 6.7 & $327.0 \pm 3.8$ & $327.2 \pm 3.7$ & $327.9 \pm 4.1$ & $305 \pm 160$ & $319.0 \pm 16.0$ \\
\hline Fi-14-07-18 & $\mathrm{X}$ & 17.97 & 153 & 114 & 0.77 & 7.3 & $287.7 \pm 5.4$ & $290.9 \pm 4.2$ & $308.1 \pm 5.1$ & $-164 \pm 850$ & $128.0 \pm 39.0$ \\
\hline Fi-14-07-19 & A & - & 159 & 87 & 0.57 & 7.0 & $326.2 \pm 3.7$ & $324.6 \pm 3.7$ & $324.8 \pm 4.0$ & $485 \pm 79$ & $341.0 \pm 12.0$ \\
\hline Fi-14-07-20 & $\mathrm{X}$ & 0.69 & 75 & 45 & 0.62 & 3.2 & $308.4 \pm 4.5$ & $310.0 \pm 4.5$ & $310.9 \pm 4.9$ & $110 \pm 240$ & $283.0 \pm 20.0$ \\
\hline Fi-14-07-24 & $\mathrm{X}$ & 2.31 & 491 & 139 & 0.29 & 23.6 & $342.1 \pm 4.2$ & $341.5 \pm 4.2$ & $342.5 \pm 4.4$ & $402 \pm 120$ & $335.0 \pm 24.0$ \\
\hline Fi-14-07-25 & $\mathrm{D}$ & - & 525 & 194 & 0.38 & 24.2 & $336.6 \pm 3.0$ & $335.9 \pm 3.0$ & $336.0 \pm 3.2$ & $405 \pm 36$ & $346.1 \pm 7.2$ \\
\hline Fi-14-07-27 & B & 0.09 & 312 & 154 & 0.51 & 14.8 & $345.6 \pm 3.3$ & $345.7 \pm 3.3$ & $345.8 \pm 3.6$ & $339 \pm 48$ & $343.1 \pm 7.7$ \\
\hline Fi-14-07-29 & Mix & 0.30 & 675 & 52 & 0.08 & 30.8 & $332.9 \pm 2.9$ & $333.4 \pm 2.9$ & $333.4 \pm 2.9$ & $281 \pm 42$ & $290.0 \pm 26.0$ \\
\hline Fi-14-07-30 & B & - & 459 & 128 & 0.29 & 21.6 & $343.6 \pm 3.1$ & $343.3 \pm 3.1$ & $343.4 \pm 3.2$ & $382 \pm 35$ & $348.7 \pm 8.0$ \\
\hline Fi-14-07-31 & B & 0.09 & 685 & 225 & 0.34 & 32.2 & $343.5 \pm 2.9$ & $343.4 \pm 3.0$ & $343.9 \pm 3.1$ & $357 \pm 29$ & $335.3 \pm 6.0$ \\
\hline Fi-14-07-32 & $\mathrm{D}$ & 0.06 & 646 & 222 & 0.35 & 29.5 & $333.7 \pm 2.9$ & $333.4 \pm 2.9$ & $334.9 \pm 3.2$ & $356 \pm 29$ & $312.0 \pm 14.0$ \\
\hline Fi-14-07-33 & B & 0.05 & 502 & 110 & 0.23 & 23.5 & $341.5 \pm 3.0$ & $341.7 \pm 3.0$ & $342.1 \pm 3.1$ & $327 \pm 34$ & $326.1 \pm 8.4$ \\
\hline Fi-14-07-34 & $\mathrm{D}$ & 0.04 & 397 & 116 & 0.30 & 18.0 & $332.5 \pm 3.0$ & $332.6 \pm 3.0$ & $332.1 \pm 3.1$ & $316 \pm 38$ & $340.9 \pm 7.8$ \\
\hline Fi-14-07-35 & $\mathrm{D}$ & 0.08 & 499 & 179 & 0.37 & 23.1 & $337.4 \pm 3.0$ & $338.0 \pm 3.0$ & $337.6 \pm 3.2$ & $279 \pm 35$ & $335.0 \pm 6.6$ \\
\hline Fi-14-07-36 & $\mathrm{D}$ & 0.08 & 370 & 269 & 0.75 & 15.9 & $315.1 \pm 3.0$ & $314.7 \pm 3.0$ & $315.4 \pm 3.4$ & $355 \pm 55$ & $312.1 \pm 5.4$ \\
\hline Fi-14-07-37 & Mix & 0.53 & 594 & 29 & 0.05 & 26.9 & $329.2 \pm 2.9$ & $329.3 \pm 3.0$ & $329.9 \pm 3.0$ & $318 \pm 51$ & $235.0 \pm 52.0$ \\
\hline Fi-14-07-38 & $\mathrm{D}$ & 0.24 & 214 & 125 & 0.60 & 9.6 & $327.0 \pm 3.3$ & $327.5 \pm 3.3$ & $326.8 \pm 3.7$ & $274 \pm 59$ & $328.9 \pm 7.8$ \\
\hline Fi-14-07-39 & B & - & 758 & 234 & 0.32 & 35.8 & $345.2 \pm 2.9$ & $345.1 \pm 2.9$ & $345.3 \pm 3.0$ & $351 \pm 24$ & $342.1 \pm 5.5$ \\
\hline Fi-14-07-40 & $\mathrm{B}$ & 0.11 & 763 & 464 & 0.63 & 35.9 & $343.9 \pm 2.9$ & $343.9 \pm 2.9$ & $345.3 \pm 3.2$ & $348 \pm 29$ & $330.2 \pm 4.7$ \\
\hline Fi-14-07-41 & $\mathrm{X}$ & 0.90 & 1518 & 591 & 0.40 & 71.9 & $342.8 \pm 2.8$ & $342.3 \pm 2.8$ & $342.2 \pm 3.0$ & $388 \pm 34$ & $352.5 \pm 6.8$ \\
\hline Fi-14-07-42 & $\mathrm{C}$ & 0.14 & 1534 & 311 & 0.21 & 78.8 & $373.7 \pm 3.0$ & $374.2 \pm 3.1$ & $374.2 \pm 3.1$ & $331 \pm 21$ & $360.1 \pm 8.1$ \\
\hline Fi-14-07-43 & $\mathrm{X}$ & 0.82 & 1089 & 336 & 0.32 & 50.8 & $338.0 \pm 2.8$ & $338.1 \pm 2.8$ & $338.9 \pm 2.9$ & $330 \pm 41$ & $320.4 \pm 8.4$ \\
\hline Fi-14-07-44 & $\mathrm{C}$ & 0.03 & 1455 & 823 & 0.58 & 69.3 & $347.9 \pm 2.8$ & $347.9 \pm 2.9$ & $348.8 \pm 3.1$ & $351 \pm 18$ & $338.6 \pm 3.8$ \\
\hline Fi-14-07-45 & $\mathrm{B}$ & 0.01 & 596 & 171 & 0.30 & 28.0 & $342.6 \pm 2.9$ & $342.4 \pm 3.0$ & $342.8 \pm 3.1$ & $360 \pm 28$ & $339.1 \pm 6.2$ \\
\hline Fi-14-07-47 & $\mathrm{C}$ & - & 224 & 109 & 0.50 & 10.5 & $342.5 \pm 3.4$ & $341.6 \pm 3.4$ & $342.3 \pm 3.7$ & $427 \pm 51$ & $345.0 \pm 10.0$ \\
\hline Fi-14-07-48 & $\mathrm{C}$ & 0.13 & 5338 & 1071 & 0.21 & 263.0 & $359.4 \pm 2.8$ & $359.5 \pm 2.8$ & $359.4 \pm 2.9$ & $351 \pm 11$ & $359.7 \pm 4.3$ \\
\hline
\end{tabular}

Errors are $1 \sigma ; \mathrm{Pb}_{\mathrm{c}}$ and $\mathrm{Pb}^{*}$ indicate the common and radiogenic portions, respectively.

Error in standard calibration was $0.20 \%$ (not included in above errors but required when comparing data from different mounts). Int: genetic interpretation of measured spot (see text of futher explanation)

(1) Common $\mathrm{Pb}$ corrected using measured ${ }^{204} \mathrm{~Pb}$ (2) Common $\mathrm{Pb}$ corrected by assuming ${ }^{206} \mathrm{~Pb} /{ }^{238} \mathrm{U}-{ }^{207} \mathrm{~Pb} /{ }^{235} \mathrm{U}$ age-concordance

(3) Common $\mathrm{Pb}$ corrected by assuming ${ }^{206} \mathrm{~Pb} /{ }^{238} \mathrm{U} \_{ }^{208} \mathrm{~Pb} /{ }^{232} \mathrm{Th}$ age-concordance 
Tab. 2 Continued

\begin{tabular}{|c|c|c|c|c|c|c|c|}
\hline $\begin{array}{c}\% \\
\text { Discordant }\end{array}$ & $\begin{array}{c}\text { Total } \\
{ }^{238} \mathrm{U} /{ }^{206} \mathrm{~Pb} \pm \%\end{array}$ & $\begin{array}{c}\text { Total } \\
{ }^{207} \mathrm{~Pb} / 206 \mathrm{~Pb} \pm \%\end{array}$ & $\begin{array}{c}(1) \\
{ }^{238} \mathrm{U}^{206} \mathrm{~Pb}^{*} \pm \%\end{array}$ & $\begin{array}{c}(1) \\
{ }^{207} \mathrm{~Pb}^{*} / 206 \mathrm{~Pb}^{*} \pm \%\end{array}$ & $\begin{array}{c}(1) \\
{ }^{207} \mathrm{~Pb}^{*} / 235 \mathrm{U} \pm \%\end{array}$ & $\begin{array}{c}(1) \\
{ }^{206} \mathrm{~Pb}^{*} / 238 \mathrm{U} \pm \%\end{array}$ & $\begin{array}{l}\text { err } \\
\text { corr }\end{array}$ \\
\hline-13 & $19.52 \pm 1.30$ & $0.0581 \pm 3.3$ & $19.67 \pm 1.40$ & $0.0518 \pm 6.0$ & $0.3630 \pm 6.2$ & $0.0508 \pm 1.4$ & 0.222 \\
\hline 36 & $19.61 \pm 1.00$ & $0.0543 \pm 2.1$ & $19.57 \pm 1.00$ & $0.0556 \pm 2.6$ & $0.3920 \pm 2.8$ & $0.0511 \pm 1.0$ & 0.367 \\
\hline-81 & $19.44 \pm 1.70$ & $0.0524 \pm 4.7$ & $19.57 \pm 1.70$ & $0.0472 \pm 7.4$ & $0.3330 \pm 7.6$ & $0.0511 \pm 1.7$ & 0.226 \\
\hline-135 & $19.46 \pm 1.10$ & $0.0756 \pm 2.3$ & $20.25 \pm 1.30$ & $0.0440 \pm 13.0$ & $0.3000 \pm 13.0$ & $0.0494 \pm 1.3$ & 0.101 \\
\hline-5 & $19.33 \pm 1.20$ & $0.0529 \pm 2.7$ & $19.34 \pm 1.20$ & $0.0525 \pm 2.9$ & $0.3740 \pm 3.1$ & $0.0517 \pm 1.2$ & 0.372 \\
\hline-72 & $22.20 \pm 1.20$ & $0.0908 \pm 2.3$ & $23.46 \pm 1.40$ & $0.0475 \pm 15.0$ & $0.2790 \pm 15.0$ & $0.0426 \pm 1.4$ & 0.097 \\
\hline-1 & $17.86 \pm 0.91$ & $0.0532 \pm 1.8$ & $17.85 \pm 0.91$ & $0.0534 \pm 1.8$ & $0.4125 \pm 2.0$ & $0.0560 \pm 0.9$ & 0.444 \\
\hline 2 & $21.32 \pm 1.00$ & $0.0714 \pm 2.6$ & $21.85 \pm 1.10$ & $0.0522 \pm 7.4$ & $0.3290 \pm 7.5$ & $0.0458 \pm 1.1$ & 0.151 \\
\hline-26 & $21.50 \pm 1.20$ & $0.0638 \pm 2.8$ & $21.87 \pm 1.30$ & $0.0504 \pm 9.3$ & $0.3180 \pm 9.4$ & $0.0457 \pm 1.3$ & 0.143 \\
\hline-38 & $19.20 \pm 1.20$ & $0.0537 \pm 3.1$ & $19.28 \pm 1.30$ & $0.0502 \pm 4.6$ & $0.3590 \pm 4.7$ & $0.0519 \pm 1.3$ & 0.267 \\
\hline-2 & $10.54 \pm 0.83$ & $0.0594 \pm 0.7$ & $10.54 \pm 0.83$ & $0.0592 \pm 0.8$ & $0.7739 \pm 1.2$ & $0.0949 \pm 0.8$ & 0.724 \\
\hline-3 & $19.64 \pm 1.00$ & $0.0557 \pm 2.0$ & $19.72 \pm 1.00$ & $0.0525 \pm 3.3$ & $0.3670 \pm 3.5$ & $0.0507 \pm 1.0$ & 0.293 \\
\hline-35 & $19.44 \pm 1.20$ & $0.0536 \pm 3.5$ & $19.52 \pm 1.20$ & $0.0503 \pm 4.8$ & $0.3550 \pm 5.0$ & $0.0512 \pm 1.2$ & 0.244 \\
\hline 4 & $18.57 \pm 0.97$ & $0.0528 \pm 1.8$ & $18.55 \pm 0.97$ & $0.0535 \pm 2.0$ & $0.3980 \pm 2.3$ & $0.0539 \pm 1.0$ & 0.430 \\
\hline-33 & $18.72 \pm 0.98$ & $0.0541 \pm 1.8$ & $18.80 \pm 0.99$ & $0.0506 \pm 3.0$ & $0.3710 \pm 3.1$ & $0.0532 \pm 1.0$ & 0.319 \\
\hline-2 & $18.65 \pm 0.97$ & $0.0527 \pm 1.8$ & $18.64 \pm 0.97$ & $0.0530 \pm 1.8$ & $0.3923 \pm 2.1$ & $0.0537 \pm 1.0$ & 0.470 \\
\hline-41 & $19.30 \pm 1.30$ & $0.0532 \pm 3.4$ & $19.37 \pm 1.40$ & $0.0499 \pm 6.3$ & $0.3550 \pm 6.4$ & $0.0516 \pm 1.4$ & 0.213 \\
\hline-13 & $18.33 \pm 0.95$ & $0.0541 \pm 1.7$ & $18.37 \pm 0.96$ & $0.0523 \pm 2.5$ & $0.3920 \pm 2.7$ & $0.0544 \pm 1.0$ & 0.353 \\
\hline 79 & $20.49 \pm 1.20$ & $0.0538 \pm 3.0$ & $20.37 \pm 1.30$ & $0.0586 \pm 5.1$ & $0.3970 \pm 5.3$ & $0.0491 \pm 1.3$ & 0.240 \\
\hline-5 & $19.62 \pm 1.20$ & $0.0525 \pm 2.8$ & $19.62 \pm 1.20$ & $0.0525 \pm 2.8$ & $0.3690 \pm 3.1$ & $0.0510 \pm 1.2$ & 0.385 \\
\hline-21 & $19.67 \pm 1.10$ & $0.0653 \pm 2.2$ & $20.02 \pm 1.20$ & $0.0512 \pm 7.1$ & $0.3520 \pm 7.2$ & $0.0500 \pm 1.2$ & 0.162 \\
\hline-7 & $19.12 \pm 1.10$ & $0.0568 \pm 2.5$ & $19.22 \pm 1.20$ & $0.0525 \pm 6.9$ & $0.3760 \pm 7.0$ & $0.0520 \pm 1.2$ & 0.172 \\
\hline-157 & $17.97 \pm 1.10$ & $0.1889 \pm 3.4$ & $21.91 \pm 1.90$ & $0.0430 \pm 34.0$ & $0.2710 \pm 34.0$ & $0.0456 \pm 1.9$ & 0.056 \\
\hline 49 & $19.35 \pm 1.10$ & $0.0533 \pm 2.6$ & $19.27 \pm 1.20$ & $0.0568 \pm 3.6$ & $0.4070 \pm 3.8$ & $0.0519 \pm 1.2$ & 0.306 \\
\hline-64 & $20.27 \pm 1.40$ & $0.0538 \pm 5.4$ & $20.41 \pm 1.50$ & $0.0482 \pm 10.0$ & $0.3260 \pm 10.0$ & $0.0490 \pm 1.5$ & 0.145 \\
\hline 37 & $17.01 \pm 0.90$ & $0.0931 \pm 0.9$ & $17.82 \pm 0.95$ & $0.0568 \pm 4.6$ & $0.4390 \pm 4.7$ & $0.0561 \pm 1.0$ & 0.203 \\
\hline-1 & $19.22 \pm 0.88$ & $0.0540 \pm 1.2$ & $19.25 \pm 0.88$ & $0.0529 \pm 1.5$ & $0.3790 \pm 1.7$ & $0.0520 \pm 0.9$ & 0.518 \\
\hline 17 & $17.92 \pm 1.20$ & $0.0733 \pm 2.9$ & $18.35 \pm 1.30$ & $0.0547 \pm 5.6$ & $0.4110 \pm 5.7$ & $0.0545 \pm 1.3$ & 0.220 \\
\hline 20 & $18.67 \pm 0.90$ & $0.0540 \pm 1.4$ & $18.66 \pm 0.91$ & $0.0548 \pm 1.6$ & $0.4052 \pm 1.9$ & $0.0536 \pm 0.9$ & 0.488 \\
\hline-2 & $18.14 \pm 0.98$ & $0.0540 \pm 1.8$ & $18.16 \pm 0.98$ & $0.0532 \pm 2.1$ & $0.4042 \pm 2.3$ & $0.0551 \pm 1.0$ & 0.422 \\
\hline-16 & $18.81 \pm 0.88$ & $0.0543 \pm 1.2$ & $18.87 \pm 0.88$ & $0.0519 \pm 1.8$ & $0.3792 \pm 2.0$ & $0.0530 \pm 0.9$ & 0.432 \\
\hline 11 & $18.28 \pm 0.92$ & $0.0537 \pm 1.5$ & $18.26 \pm 0.92$ & $0.0543 \pm 1.6$ & $0.4097 \pm 1.8$ & $0.0548 \pm 0.9$ & 0.508 \\
\hline 4 & $18.25 \pm 0.87$ & $0.0544 \pm 1.2$ & $18.27 \pm 0.87$ & $0.0537 \pm 1.3$ & $0.4050 \pm 1.6$ & $0.0547 \pm 0.9$ & 0.561 \\
\hline 7 & $18.81 \pm 0.88$ & $0.0541 \pm 1.2$ & $18.82 \pm 0.88$ & $0.0536 \pm 1.3$ & $0.3928 \pm 1.6$ & $0.0531 \pm 0.9$ & 0.564 \\
\hline-4 & $18.37 \pm 0.90$ & $0.0534 \pm 1.4$ & $18.38 \pm 0.90$ & $0.0530 \pm 1.5$ & $0.3973 \pm 1.8$ & $0.0544 \pm 0.9$ & 0.510 \\
\hline-5 & $18.89 \pm 0.92$ & $0.0531 \pm 1.5$ & $18.89 \pm 0.92$ & $0.0527 \pm 1.7$ & $0.3846 \pm 1.9$ & $0.0529 \pm 0.9$ & 0.484 \\
\hline-17 & $18.59 \pm 0.90$ & $0.0525 \pm 1.4$ & $18.61 \pm 0.90$ & $0.0519 \pm 1.5$ & $0.3842 \pm 1.8$ & $0.0537 \pm 0.9$ & 0.505 \\
\hline 13 & $19.95 \pm 0.96$ & $0.0543 \pm 2.2$ & $19.96 \pm 0.96$ & $0.0536 \pm 2.4$ & $0.3704 \pm 2.6$ & $0.0501 \pm 1.0$ & 0.367 \\
\hline-3 & $18.98 \pm 0.91$ & $0.0570 \pm 1.2$ & $19.09 \pm 0.91$ & $0.0528 \pm 2.2$ & $0.3811 \pm 2.4$ & $0.0524 \pm 0.9$ & 0.377 \\
\hline-16 & $19.17 \pm 1.00$ & $0.0537 \pm 2.1$ & $19.22 \pm 1.00$ & $0.0517 \pm 2.6$ & $0.3710 \pm 2.8$ & $0.0520 \pm 1.0$ & 0.369 \\
\hline 2 & $18.19 \pm 0.86$ & $0.0533 \pm 1.1$ & $18.18 \pm 0.86$ & $0.0535 \pm 1.1$ & $0.4058 \pm 1.4$ & $0.0550 \pm 0.9$ & 0.626 \\
\hline 1 & $18.23 \pm 0.86$ & $0.0544 \pm 1.1$ & $18.25 \pm 0.86$ & $0.0535 \pm 1.3$ & $0.4039 \pm 1.6$ & $0.0548 \pm 0.9$ & 0.553 \\
\hline 13 & $18.15 \pm 0.82$ & $0.0616 \pm 0.7$ & $18.31 \pm 0.83$ & $0.0544 \pm 1.5$ & $0.4098 \pm 1.7$ & $0.0546 \pm 0.8$ & 0.476 \\
\hline-11 & $16.73 \pm 0.83$ & $0.0542 \pm 0.7$ & $16.75 \pm 0.83$ & $0.0531 \pm 0.9$ & $0.4365 \pm 1.2$ & $0.0597 \pm 0.8$ & 0.670 \\
\hline-2 & $18.42 \pm 0.84$ & $0.0596 \pm 0.9$ & $18.58 \pm 0.84$ & $0.0530 \pm 1.8$ & $0.3936 \pm 2.0$ & $0.0538 \pm 0.8$ & 0.423 \\
\hline 1 & $18.03 \pm 0.83$ & $0.0537 \pm 0.8$ & $18.03 \pm 0.83$ & $0.0535 \pm 0.8$ & $0.4092 \pm 1.2$ & $0.0555 \pm 0.8$ & 0.716 \\
\hline 5 & $18.32 \pm 0.88$ & $0.0538 \pm 1.2$ & $18.32 \pm 0.88$ & $0.0537 \pm 1.2$ & $0.4044 \pm 1.5$ & $0.0546 \pm 0.9$ & 0.581 \\
\hline 25 & $18.35 \pm 1.00$ & $0.0542 \pm 2.0$ & $18.33 \pm 1.00$ & $0.0554 \pm 2.3$ & $0.4170 \pm 2.5$ & $0.0546 \pm 1.0$ & 0.407 \\
\hline-2 & $17.42 \pm 0.80$ & $0.0546 \pm 0.4$ & $17.44 \pm 0.80$ & $0.0535 \pm 0.5$ & $0.4232 \pm 1.0$ & $0.0573 \pm 0.8$ & 0.843 \\
\hline
\end{tabular}


convolute or patchy zoning, gave $\mathrm{U}-\mathrm{Pb}$ ages that spread between magmatic and anatectic age estimates. This result lends support to the interpretation of these domains as incompletely modified during anatexis, through recrystallization and/or localized reprecipitation.

\section{Discussion and conclusions}

According to the results of this SHRIMP study, the magmatic protolith of the Hbl-bearing diatexite from TittlingWaldkirchen crystallized at $343.9 \pm 2.3 \mathrm{Ma}$. Around this time, there was a considerable I-type plutonic activity in the central part of the Bohemian Massif (Fig. 2), culminating in the formation of the large Central Bohemian Plutonic Complex (Holub et al. 1997a; Janoušek et al. 2000b). We suggest that the Tittling-Waldkirchen orthodiatexite can be correlated with this complex for the following two reasons:

1) A characteristic feature of the central Bohemian I-type plutonic terrain is the coeval emplacement of felsic and mafic magma, resulting in a widespread presence of mafic enclaves and dikes within granitoids. Magma mingling textures, such as those described by Janoušek et al. (2010), are comparable to enclave-host relationships in the Tittling-Waldkirchen diatexite (Fig. 3).

2) The Central Bohemian Plutonic Complex includes a variety of intrusive lithologies (Holub et al. 1997b; Žák et al. 2005; Janoušek et al. 2010), including the c. $354 \mathrm{Ma}$, medium-K, mostly tonalitic Sázava suite (Janoušek et al. 1995, 2004), the c. 345 Ma high-K, granodioritic Blatná suite (Janoušek et al. 2010), and the c. $340 \mathrm{Ma}$ high-K granodioritic to syenitic Čertovo břemeno suite (Holub 1997; Holub et al. 1997a). Geochemically, the diatexite from Tittling-Waldkirchen has many similarities with the Červená granodiorite (René 1999), a member of the Blatná suite. These similarities include $\mathrm{SiO}_{2}$ content (mainly 60-65 wt \%), a high-K, calc-alkaline metaluminous to slightly peraluminous $(\mathrm{A} / \mathrm{CNK}=0.9-1.1)$ composition and high $\mathrm{CaO}$, consistent with the presence of hornblende. Trace-element contents, including the REE, are also comparable (Tab. 1). Notably, our age estimate for the Tittling-Waldkirchen ortho-diatexite corresponds exactly with that of the Blatná suite.

It has been speculated in previous work that certain geological formations continue from central Bohemia southwestwards into the Bavarian Zone, where they were reworked by late Variscan (post-330 Ma) orogenesis and anatexis (Finger et al. 2007). One such lithology is the so-called 'palite', an ortho-diatexite near the Pfahl Fault (Fig. 2), that has been correlated with potassic ('durbachite') plutons to the northeast, including the Čertovo bremeno suite of the Central Bohemian Plutonic
Complex. Other examples are granulites and graphitebearing gneisses in the Kropfmühl Unit north of Passau ("K" in Fig. 2), which are likely to correspond to similar lithologies near Český Krumlov, north of the Bavarian Zone. The ortho-diatexite from Tittling-Waldkirchen provides another unifying feature between the Bavarian Zone and the central Bohemian Massif. Such correlations work against tectonic models suggesting that the Bavarian Zone is a crustal terrane independent of the central Bohemian Massif, with which it was juxtaposed along the Pfahl Fault (Siebel et al. 2008, 2009).

Relationships between lithological sequences in the Bavarian Zone and the central Bohemian Massif are greatly obscured by crustal movements during the Bavarian Phase of orogenesis, for which little kinematic data have been obtained. In view of the dextral movement along the Pfahl Fault (e.g., Verner et al. 2008, GaladíEnríquez et al. 2010), the Tittling-Waldkirchen orthodiatexite cannot be traced back to the south-western end of the Central Bohemian Plutonic Complex, but would rather have been emplaced as a discrete plutonic body further southeast. Considering the chemical and age equivalence, we presume that this pluton and the Central Bohemian Plutonic Complex occurred in tectonically equivalent positions, thus tracing the western flank of the Moravo-Moldanubian Fold Belt (Finger et al. 2007) or, in terms of the alternative model, tracing the magmatic arc of the Teplá-Barrandian active plate margin (Žák et al. 2005, 2010). Both models allow for a role of the more western parts of the Bavarian Zone as a reworked southern continuation of the Teplá-Barrandian Unit, a possibility that can readily be tested by detailed geochronological work in future studies.

Acknowledgements. We thank Kenneth Johnson, Milan Kohút and Jiří Žák for their constructive reviews.

\section{References}

Black LP, Kamo SL, Allen CM, Davis DW, Aleinikoff JN, Valley JW, Mundilf R, Campbell IH, Korscha IJ, Williams IS, Foudoulis C (2004) Improved ${ }^{206} \mathrm{~Pb} /{ }^{238} \mathrm{U}$ microprobe geochronology by the monitoring of a trace-element-related matrix effect; SHRIMP, ID-TIMS, ELA-ICP-MS and oxygen isotope documentation for a series of zircon standards. Chem Geol 205: 115-140

Corfu F, Hanchar JM, Hoskin PWO, Kinny P (2003) Atlas of zircon textures. In: HANCHAR JM, HOSKIN PWO (eds) Zircon. Mineralogical Society of America and Geochemical Society, Reviews in Mineralogy and Geochemistry 53: 469-500

Dallmeyer RD, Franke W, Weber K (1995) Pre-Permian Geology of Central and Eastern Europe. Springer, Berlin, pp 1-604 
Edel JB, Schulmann K, Holub FV (2003) Anticlockwise rotations of the Eastern Variscides accommodated by dextral lithospheric wrenching: paleomagnetic and structural evidence. J Geol Soc, London 160: 209-218

Fiala J, Fuchs G, Wendt JI (1995) Stratigraphy of the Moldanubian Zone. In: Dallmeyer RD, Franke W, WeBER K (eds) Pre-Permian Geology of Central and Eastern Europe. Springer, Berlin, pp 417-428

Finger F, Steyrer HP (1995) A tectonic model for the eastern Variscides: indications from a chemical study of amphibolites in the south-eastern Bohemian Massif. Geol Carpath 46: 137-150

Finger F, Gerdes A, Janoušek V, René M, Riegler G (2007) Resolving the Variscan evolution of the Moldanubian sector of the Bohemian Massif: the significance of the Bavarian and the Moravo-Moldanubian tectonometamorphic phases. J Geosci 52: 9-28

Finger F, Gerdes A, René M, Riegler G (2009) The SaxoDanubian Granite Belt: magmatic response to postcollisional delamination of mantle lithosphere below the south-western sector of the Bohemian Massif (Variscan Orogen). Geol Carpath 60: 205-212

Finger F, René M (2009) A comment on 'Two distinctive granite suites in the SW Bohemian Massif and their record of emplacement: constraints from geochemistry and zircon ${ }^{207} \mathrm{~Pb} /{ }^{206} \mathrm{~Pb}$ chronology' by Siebel et al., Journal of Petrology 49:1853-1872. J Petrol 50: 591-593

FisCHER G (1967) Über das Moldanubikum der Bayerischen Oberpfalz und des Bayerischen Waldes. Aufschluß 18: $27-111$

Franke W (1989) Tectonostratigraphic units in the Variscan belt of central Europe. In: DALLMEYER RD (ed) Terranes in Circum-Atlantic Paleozoic Orogens. Geological Society of America Special Papers 230: 67-90

Franke W (2000) The middle-European segment of the Variscides: tectonostratigraphic units, terrane boundaries and plate tectonic evolution. In: Franke W, HaAK U, Oncken O, TANner D (eds) Orogenic Processes: Quantification and Modelling in the Variscan Belt. Geological Society of London Special Publications 179: 35-61

Franke W, ŻelaźNIEWICZ A (2002) Structure and evolution of the Bohemian Arc. In: Winchester JA Pharaoh TC, Verniers J (eds) Palaeozoic Amalgamation of Central Europe. Geological Society of London Special Publications 201: 279-293

Friedl G, Finger F, McNaughton N, Fletcher IR (2000) Deducing the ancestry of terranes: SHRIMP evidence for South America-derived Gondwana fragments in Central Europe. Geology 28: 1035-1038

Fritz H, Neubauer F (1993) Kinematics of crustal stacking and dispersion in the south-eastern Bohemian Massif. Geol Rundsch 82: 556-565

Fuchs G (1976) Zur Entwicklung der Böhmischen Masse. Jb Geol B-A 129: 41-49
Galadí-Enríquez E, Dörr W, Zulauf G, Galindo-Zaldívar J, Heidelbach F, Rohrmüller J (2010) Variscan deformation phases in the southwestern Bohemian Massif: new constraints from sheared granitoids. Z Dt Ges Geowiss 161: 1-23

Harley SL, Kelly NM, Möller A (2007) Zircon behaviour and the thermal histories of mountain chains. Elements 3: $25-30$

Holub FV (1997) Ultrapotassic plutonic rocks of the durbachite series in the Bohemian Massif: petrology, geochemistry, and petrogenetic interpretation. Sbor geol věd, ložisk Geol Mineral 31: 5-26

Holub FV, Cocherie A, Rossi P (1997a) Radiometric dating of granitic rocks from the Central Bohemian Plutonic Complex: constraints on the chronology of thermal and tectonic events along the Barrandian-Moldanubian boundary. C R Acad Sci Paris, Sciences de la Terre et des planétes 325: 19-26

Holub FV, Machart J, Manová M (1997b) The Central Bohemian Plutonic Complex: geology, chemical composition and genetic interpretation. Sbor geol věd, ložisk Geol Mineral 31: 27-50

JaNoušek V, Rogers G, Bowes DR, (1995) Sr-Nd isotopic constraints on the petrogenesis of the Central Bohemian Pluton, Czech Republic. Geol Rundsch 84: 520-534

Janoušek V, Bowes DR, Braithwaite CJR, Rogers G (2000a) Microstructural and mineralogical evidence for limited involvement of magma mixing in the petrogenesis of a Hercynian high-K calc-alkaline intrusion: the Kozárovice granodiorite, Central Bohemian Pluton, Czech Republic. Trans Roy Soc Edinb, Earth Sci 91: $15-26$

Janoušek V, Bowes DR, Rogers G, Farrow CM, Jelínek E (2000b) Modelling diverse processes in the petrogenesis of a composite batholith: the Central Bohemian Pluton, Central European Hercynides. J Petrol 41: 511-543

Janoušek V, Braithwaite CJR, Bowes DR, Gerdes A (2004) Magma-mixing in the genesis of Hercynian calc-alkaline granitoids: an integrated petrographic and geochemical study of the Sázava intrusion, Central Bohemian Pluton, Czech Republic. Lithos 78: 67-99

JANOUŠEK V, WIEGAND BA, ŽÁ J (2010) Dating the onset of Variscan crustal exhumation in the core of the Bohemian Massif: new $\mathrm{U}-\mathrm{Pb}$ single zircon ages from the high- $\mathrm{K}$ calc-alkaline granodiorites of the Blatná suite, Central Bohemian Plutonic Complex. J Geol Soc, London 167: 347-360

Kalt A, Corfu F, Wisbrams JR (2000) Time calibration of a $\mathrm{P}-\mathrm{T}$ path from a Variscan high-temperature low-pressure metamorphic complex (Bayerische Wald, Germany), and the detection of inherited monazite. Contrib Mineral Petrol 138: 143-163

Konopásek J, Schulmann K (2005) Contrasting Early Carboniferous field geotherms: evidence for accretion of a 
thickened orogenic root and subducted Saxothuringian crust (central European Variscides). J Geol Soc, London 162: 463-470

Ludwig KR (2001) SQUID: A User's Manual. Berkeley Geochronology Center, Special Publications 2: 1-22

Ludwig KR (2003) User's Manual for Isoplot 3.00: A Geochronological Toolkit for Microsoft Excel. Berkeley Geochronology Center, Special Publications 4: 1-71

Oncken O, Plesch A, Weber J, Ricken W, Schrader S (2000) Passive margin detachment during arc-continent collision (Central European Variscides). In: Franke W, HaAK V, Oncken O, TANner D (eds) Orogenic Processes: Quantification and Modelling in the Variscan Belt. Geological Society of London Special Publications 179: 199-216

Propach G, Kling M, Linhardt E, Rohrmüller J (2008) Reste eines Inselbogens in der Moldanubischen Zone des Bayerischen Waldes. Geol Bavarica 110: 343-377

René M (1999) Petrogenesis of granitoids of the Červená type (Central Bohemian Plutonic Complex). Acta Montana A 14: 81-97

Schulmann K, Kröner A, Hegner E, Wendt I, Konopásek J, LEXA O, ŠTíPSKÁ P (2005) Chronological constraints on the pre-orogenic history, burial and exhumation of deepseated rocks along the eastern margin of the Variscan Orogen, Bohemian Massif, Czech Republic. Amer J Sci 305: $407-448$

Schulmann K, Konopásek J, Janoušek V, Lexa O, Lardeaux J-M, Edel J-B, ŠTíPSKÁ P, Ulrich S (2009) An Andean type Palaeozoic convergence in the Bohemian Massif. C R Geoscience 341: 266-286

Siebel W, Shang C, Reitter E, Rohrmüller J, Breiter K (2008) Two distinctive granite suites in the SW Bohemian Massif and their record of emplacement: constraints from geochemistry and zircon ${ }^{207} \mathrm{~Pb} /{ }^{206} \mathrm{~Pb}$ chronology. J Petrol 49: 1853-1872

Siebel W, Shang CK, Reitter E, Rohrmüller J, Breiter K (2009) 'Two distinctive granite suites in the southwestern Bohemian Massif' - Reply to F. Finger and M. René. J Petrol 50: 595-599

SŁaby E, Martin H (2008) Mafic and felsic magma interaction in granites: the Hercynian Karkonosze Pluton (Sudetes, Bohemian Massif). J Petrol 49: 353-391

Stacey JC, Kramers J (1975) Approximation of terrestrial lead isotope evolution by a two-stage model. Earth Planet Sci Lett 26: 207-221

SteIN E (1988) Die strukturgeologische Entwicklung im Übergangsbereich Saxothuringikum/Moldanubikum in NE-Bayern. Geol Bavarica 92: 5-31

Thiele O (1962) Neue geologische Ergebnisse aus dem Sauwald (O.Ö.). Verh Geol B-A, pp 117-129
Troll G (1967) Bau und Bildungsgeschichte des Bayerischen Waldes. Geol Bavarica 58: 15-21

Tropper P, Deibl I, Finger F, Kaindl R (2006) P-T-t evolution of spinel-cordierite-garnet gneisses from the Sauwald Zone (southern Bohemian Massif, Upper Austria): is there evidence for two independent late-Variscan low-P/high-T events in the Moldanubian Unit? Int J Earth Sci 95: 1019-1037

Urban M, Synek J (1995) VII. C 2 Moldanubian Zone. Structure. In: Dallmeyer RD, Franke W, Weber K (eds) Pre-Permian Geology of Central and Eastern Europe. Springer, Berlin, pp 429-443

Verner K, Ž́́́ J, Pertoldová J, Šrámek J, Sedlák J, TrubaČ J, TÝcovÁ P (2008) Magmatic history and geophysical signature of a post-collisional intrusive center emplaced near a crustal-scale shear zone: the Plechý granite pluton (Moldanubian Batholith, Bohemian Massif). Int J Earth Sci 98: 517-532

Wiebe RA, Smith D, Sturm M, King EM, Seckler MS (1997) Enclaves in the Cadillac Mountain granite (coastal Maine): samples of hybrid magma from the base of the chamber. J Petrol 38: 393-423

Wiedenbeck M, Allé P, Corfu F, Griffin WL, Meier M, Oberli F, von Quadt A, Roddick JC, Spiegel W (1995) Three natural zircon standards for $\mathrm{U}-\mathrm{Th}-\mathrm{Pb}, \mathrm{Lu}-\mathrm{Hf}$, trace element and REE analysis. Geostand Newsl 19: 1-23

Winchester JA, Pharaoh TC, Verniers J (2002) Palaeozoic amalgamation of central Europe. An introduction and synthesis of new results from recent geological and geophysical investigations. In: Winchester JA, PHARAOH TC, Verniers J (eds) Palaeozoic Amalgamation of Central Europe. Geological Society of London Special Publications 201: 1-18

ZeH A, Gerdes A (2010) Baltica- and Gondwana-derived sediments in the Mid-German Crystalline Rise (Central Europe): implications for the closure of the Rheic Ocean. Gondwana Res 17: 254-263

Zulauf G (1997) Von der Anchizone bis zur Eklogitfazies: angekippte Krustenprofile als Folge der cadomischen und variscischen Orogenese im Teplá-Barrandium (Böhmische Masse). Geotekt Forsch 89: 1-302

ŽÁK J, Holub FV, Verner K (2005) Tectonic evolution of a continental magmatic arc from transpression in the upper crust to exhumation of mid-crustal orogenic root recorded by episodically emplaced plutons: the Central Bohemian Plutonic complex (Bohemian Massif). Int J Earth Sci 94: 385-400

Žák J, Kratinová Z, Trubač J., Janoušek V, Sláma J, MrLINA J (in print) Structure, emplacement, and tectonic setting of Late Devonian granitoid plutons in the TepláBarrandian Unit, Bohemian Massif. Int J Earth Sci, doi 10.1007/s00531-010-0565-7 